n 1856 the pathologist Virchow published his now classic observations on atherothrombosis based on the examination of postmortem tomographic artery sections. The Virchow triad describes three components contributing to the atherothrombotic disease process: the vessel wall, the blood constituents, and blood flow.

In vivo coronary imaging techniques became available more than 100 years later with the introduction of selective coronary angiography in 1958 by Mason Sones. Selective coronary angiography has allowed the identification of significantly stenotic, advanced coronary lesions and narrowed the clinical interest of cardiologists on luminal dimensions. This singular focus on angiographic stenosis has resulted in a proliferation of surgical and catheter based revascularisation techniques allowing mechanical treatment of focal coronary artery disease (CAD). Expressions like "fixing rusty pipes" and "plumbing", used by cardiologist to describe atherosclerosis and its treatment, reflect a mechanistic view of $\mathrm{CAD}$, based on the enormous success of these myocardial revascularisation techniques.

However, Virschow's observations and more contemporary models of atherosclerotic disease have led to an understanding of CAD as a systemic disease of a complex organ system (the vessel wall) and its environment (the blood components and flow phenomena). Importantly, the complex atherosclerotic disease process is frequently not reflected in the luminal silhouette because most lesions have silently developed over a long time before they obstruct the lumen.

Therefore direct imaging of the vessel wall has become a new goal in the assessment of CAD progression and prevention. Intravascular ultrasound (IVUS) represents the first clinical imaging technique enabling routine tomographic imaging of coronary arteries. Comprehensive technical and clinical reviews of IVUS have recently been published. ${ }^{12}$ In this article we will give a brief summary of IVUS techniques and then describe the role of tomographic coronary imaging for a contemporary understanding of CAD using examples from past and present experience with IVUS.

\title{
TECHNICAL CONSIDERATIONS
}

Correspondence to: Steven E Nissen, MD, The Cleveland Clinic Foundation Desk F-25, 9500 Euclid Avenue, Cleveland $\mathrm{OH}$ 44195, USA; nissens@ccf.org
IVUS is performed during cardiac catheterisation using miniature ultrasound probes mounted on the tip of a coronary catheter. The IVUS probe emits high ultrasound frequencies, typically centred at 20-50 MHz. The ultrasound signal reflected from arterial wall structures is used to generate a grey scale image. Using a $30 \mathrm{MHz}$ probe, corresponding to a wavelength of $50 \mu \mathrm{m}$, axial and lateral resolution is approximately 150 and $250 \mu \mathrm{m}$, respectively. The probe is placed beyond the target lesion site and the ultrasound catheter is then withdrawn during continuous imaging, resulting in a series of tomographic images of the vessel wall (fig 1).

Ultrasound is strongly reflected at the interface of different tissue structures. In coronary arteries these are the blood-intimal border and the external elastic membrane (EEM). Manual or computer aided planimetry of these two borders allows precise measurements of the lumen area, intima-media area, and EEM. ${ }^{1}$ In addition, the ultrasound signal backscattered from the arterial wall allows the various tissue components to be differentiated, including the atherosclerotic plaque. The visual appearance of IVUS images of atheroma describe a continuum from echodense (bright echo signal) to echolucent (faint echo signal). ${ }^{2}$ However, recent approaches using advanced image processing of the raw IVUS data such as radiofrequency analysis have improved characterisation of vessel wall characteristics.

Using IVUS, some morphologic features of atherosclerotic coronary plaques can be readily recognised. The reliability of ultrasound imaging in predicting the composition of atherosclerotic plaque components has been demonstrated in comparative studies of histology. Lipid laden lesions appear as hypoechoic, "soft" areas and fibrous or calcified tissues are recognised as bright echoes. In lipid laden lesions with prominent overlying fibrous "caps", a more reflective structure separating the soft echoes from the lumen is identified on the corresponding images (fig 2). The integration of information from adjacent images in a coronary segment allows three dimensional reconstruction and the calculation of atheroma volume ( fig l). 


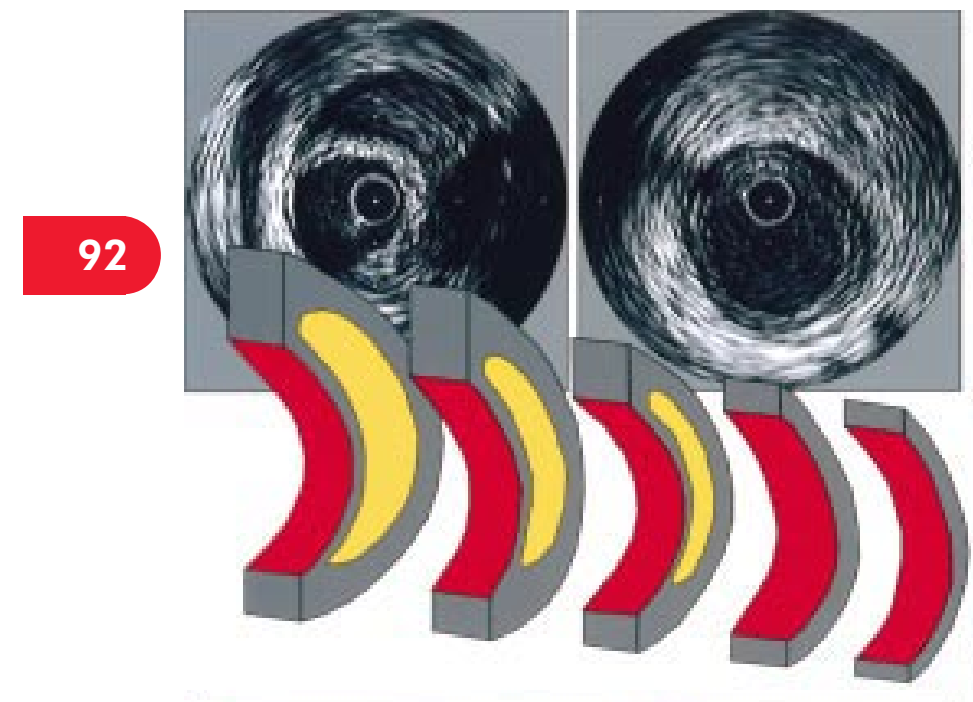

Lumen $\square$ Vessel wall $\square$ Lipid rich core

\section{NATIVE ATHEROSCLEROTIC LESIONS AND THE VESSEL WALL}

Selective coronary angiography depicts coronary arteries as a planar silhouette of the contrast filled lumen. Lesions are defined angiographically by the focal narrowing of the luminal silhouette. However, severe atherosclerosis may not lead to an apparent luminal stenosis if the atheroma affects the entire vessel segment (diffuse disease). Furthermore, as originally described by Glagov and colleagues, atheromatous disease may result in focal expansion of the vessel size (enlargement of the EEM), a process known as arterial remodelling. ${ }^{3}$ IVUS has been extraordinarily useful in confirming these two phenomena (diffuse disease and remodelling) in vivo (figs 1 and 2 ).

\section{Diffuse disease}

The experience with transplant vasculopathy is a particularly striking demonstration of the angiographic underestimation

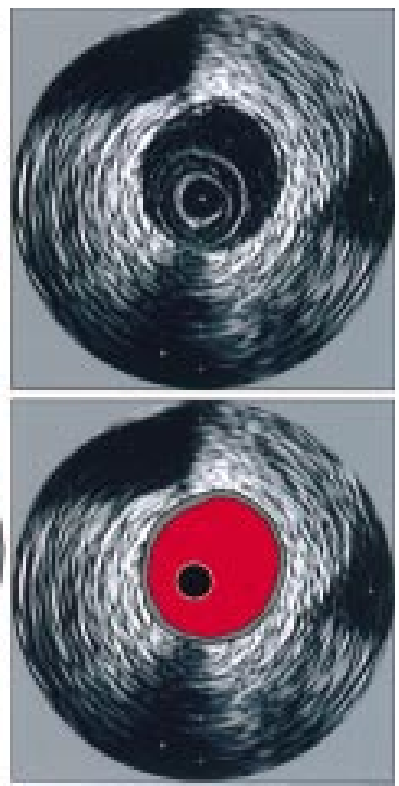

Figure 1 Principle of IVUS transducer pullback. The transducer pullback through a vessel segment allows the assessment of adjacent image slices and the three dimensional reconstruction. Importantly, tomographic imaging with IVUS allows the assessment of the coronary arterial lumen and vessel wall. Therefore the atherosclerotic plaque can be directly visualised. The first IVUS panel (top left) shows a crescent of severe atheroma extending for most of the vessel circumference from the 10 o'clock position to the 7 o'clock position. This lesion has a faint (lucent) echo signal of a lipid rich core of atheroma with a more echogenic fibrous cap.
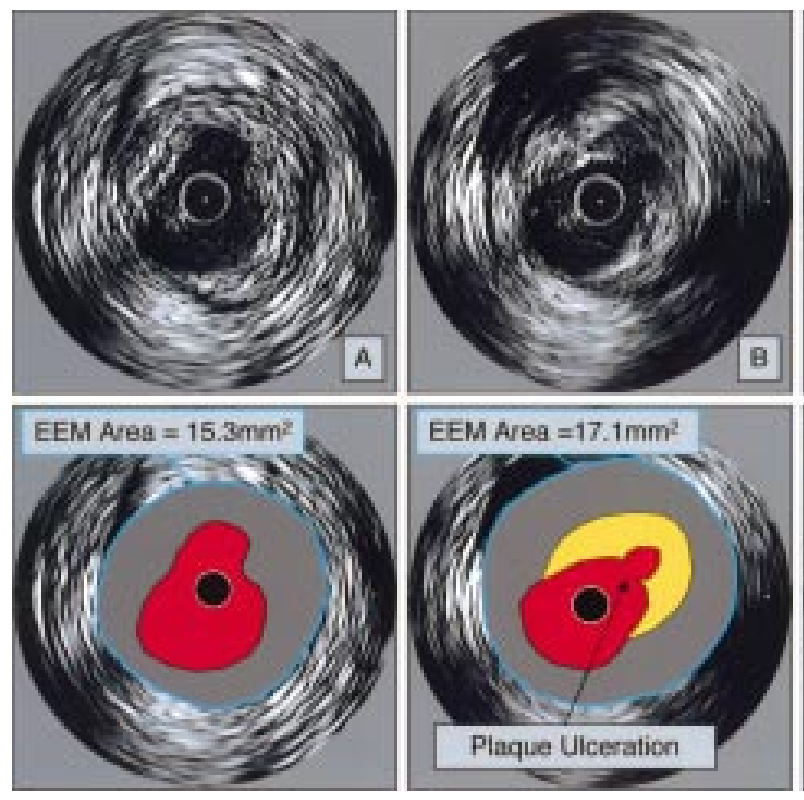

of diffuse disease. Coronary transplant vasculopathy represents the major cause of death in the first year after transplantation. However, because the heart is denervated, post-transplant coronary obstruction does not lead to angina pectoris. Therefore annual surveillance angiography, with and without IVUS, is commonly performed in patients after cardiac transplantation. Comparative IVUS studies have demonstrated the insensitivity of angiography in detecting transplant vasculopathy. Angiographic disease is present in only $10-20 \%$ of patients at one year and in $50 \%$ by five years, while the prevalence of arteriopathy detected by ultrasound is much higher, with abnormal intimal thickening seen by IVUS in $50 \%$ of patients by one year. ${ }^{4}$ The diffuse involvement of entire coronary segments, which is characteristic for transplant vasculopathy, explains why this disease process does not lead to focal luminal narrowing and therefore is frequently not detected by angiography. 

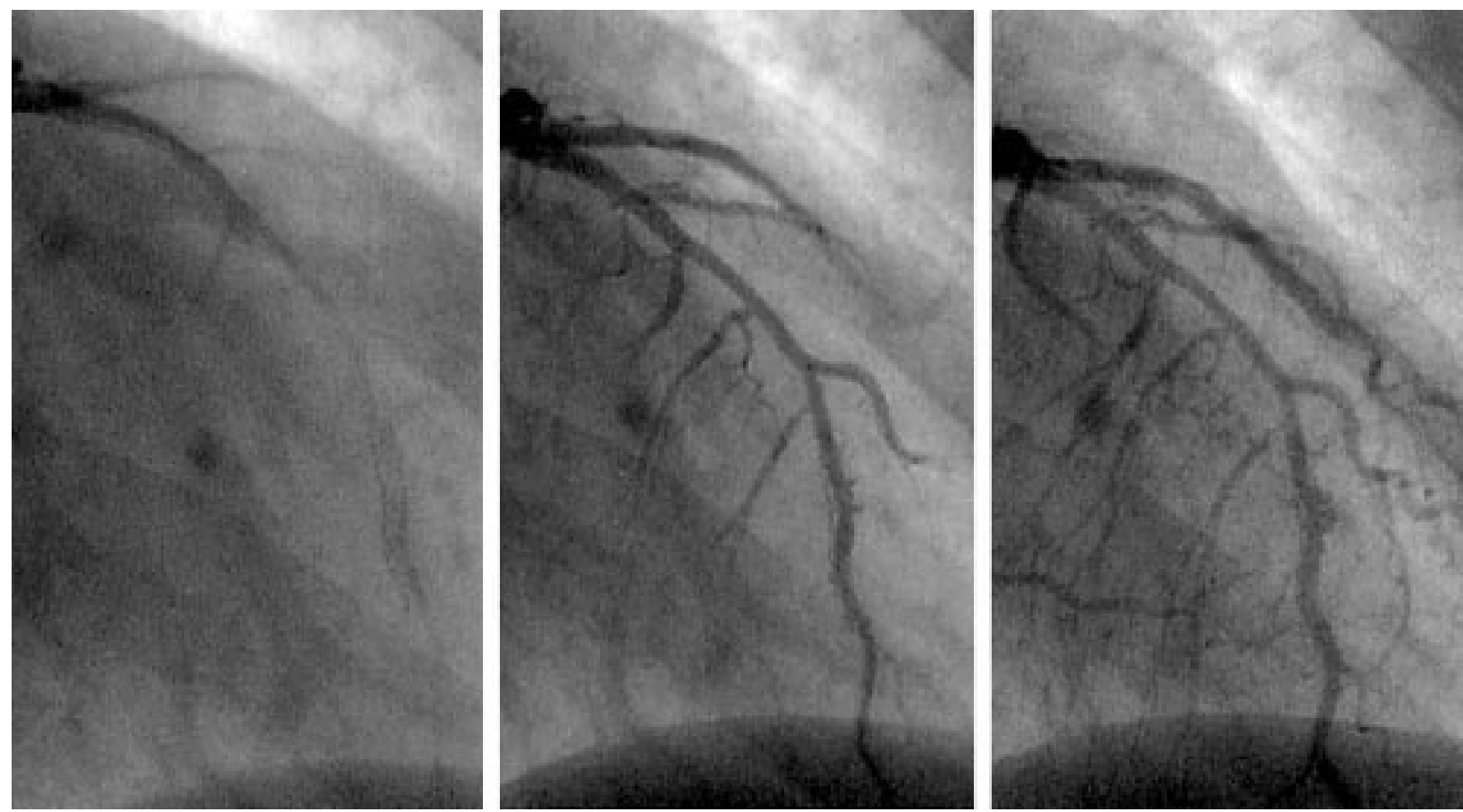

Figure 3 Clinical application of IVUS. Figures 3-5 show images obtained from a 26 year old patient, who presented three months after stent deployment in the LAD for severe CAD. This fig shows the selective coronary angiogram of the previously stented LAD lesion. A focal expansion of the lumen at the proximal stent edge is visible. The silhovette of the angiogram does not define the anatomy of such non-obstructive lesions.

A similar process may go unrecognised in typical native vessel atherosclerosis. Several IVUS studies have shown that angiographically "normal reference sites" are frequently affected by atherosclerosis." The angiographic finding of "small vessels"-for example, in diabetic patients—represents another extreme example of diffuse plaque accumulation without focal narrowing.

\section{Arterial remodelling}

Another important observation has evolved from IVUS examination of transplanted hearts. In some transplant centres, a baseline angiographic and IVUS examination of one or more coronary vessels has been performed routinely. This is typically performed within a few weeks following transplantation and thus reflects the state of the vessel at the time of donor death. Most transplant donors are young in age, typically succumbing to trauma from motor vehicle accidents. Despite the young age of these donors, significant atherosclerosis is frequently detected by IVUS in donor coronary arteries. In one published study, more than half of donors at a mean age of 32 years had at least one site with an intimal thickness exceeding $0.5 \mathrm{~mm} .{ }^{6}$ Strikingly, nearly all of these subjects had completely normal angiograms.

The finding of diffuse or focal lesions without angiographic stenosis perplexed cardiologists who viewed diseased coronary arteries as "rusty pipes". In a solid pipe, accumulation of debris should always lead to a decreased luminal diameter. However, it seems clear that this paradigm is not appropriate in coronary arteries. In 1987 the pathologist Seymour Glagov described a crucial observation about early atherosclerotic lesion development. ${ }^{3}$ Based on previous examinations in postmortem and animal models, Glagov described a positive correlation between EEM area and atheroma area in necropsy specimens of human postmortem arteries. The author hypothesised that focal disease is often not evident as luminal obstruction because of "compensatory" expansion of the vessel wall (arterial remodelling). Specifically, lesions with a stenosis $<40 \%$ were counterbalanced by an increase in arterial size that "compensated" for plaque accumulation, maintaining lumen area. In advanced lesions, remodelling was less evident and lumen size was reduced.

IVUS has allowed the in vivo study of remodelling. Ultrasound studies confirmed the correlation between EEM and plaque area and compensatory vessel enlargement or "positive remodelling" in early disease. ${ }^{7}$ Subsequent IVUS studies have demonstrated a new dimension to arterial remodelling, negative remodelling or arterial shrinkage. ${ }^{8}$ At diseased sites, the EEM may actually shrink in size, contributing to luminal stenosis. Initially described in restenotic lesions after coronary intervention, negative remodelling can also be found in mildly stenotic lesions of native coronary arteries.

Our understanding of the central role of remodelling in the pathophysiology of CAD is evolving. Initially positive remodelling was seen as merely compensatory, and therefore a welcome "positive" process. However, recent IVUS studies examining the relation between remodelling and clinical presentation in patients with CAD suggest a more complex role. In unstable patients, both EEM and plaque areas were significantly larger than the corresponding measurements in stable patients. In other words, positive remodelling is significantly more prevalent in the unstable patients and negative remodelling more prevalent in the stable patients. ${ }^{9}$ Lesion development and, in particular, lesion stability appear to be related to the direction of arterial remodeling.

The initial experience with IVUS showed that the simultaneous assessment of lumen and vessel wall could provide important insights into lesion development and significance. It was soon discovered that these principles also apply to lesion response during coronary interventions (figs 3, 4, and 5). 

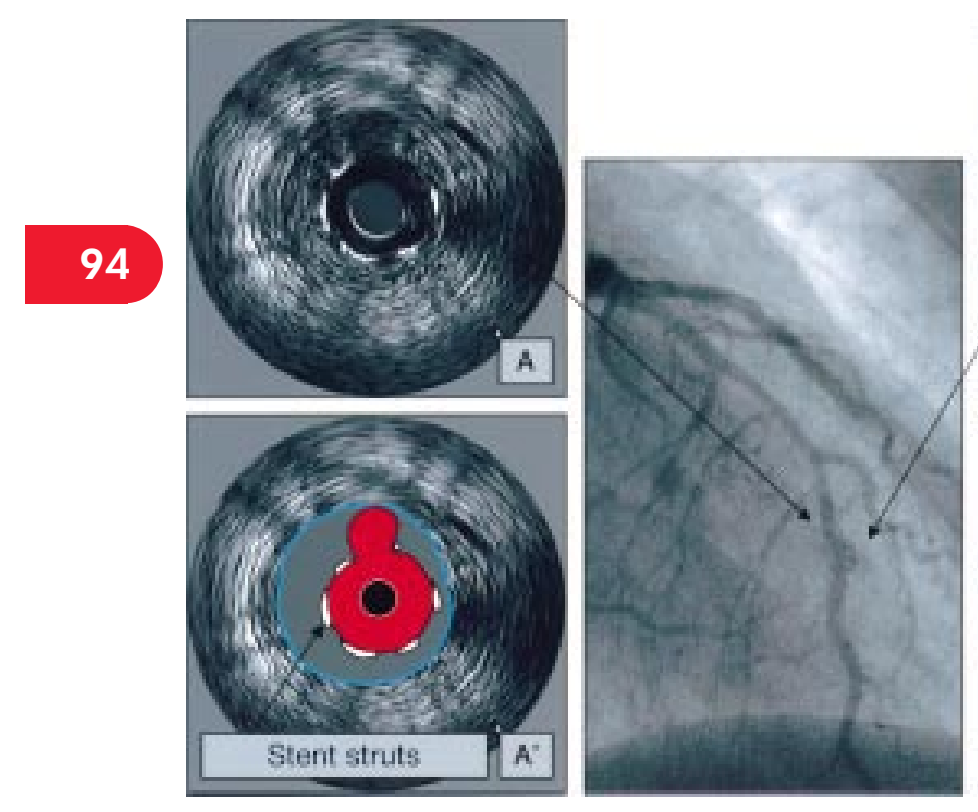

\section{PERCUTANEOUS CORONARY INTERVENTIONS}

\section{Restenosis}

IVUS has shaped our understanding of restenosis after coronary percutaneous intervention. Initially, investigators believed that the predominant mechanism of restenosis after balloon angioplasty was intimal proliferation. However, ultrasound studies in peripheral vessels provided evidence that negative remodelling, or localised shrinkage of the vessel, was a major mechanism of late lumen loss. ${ }^{8}$ Mintz and colleagues studied 212 native coronary arteries in patients undergoing repeat catheterisation after coronary interventions. At follow up, there was a decrease in EEM area and an increase in plaque area at the target lesion. ${ }^{10}$ Interestingly, more than $70 \%$ of lumen loss was attributable to the decrease in EEM area, whereas the neointimal growth accounted for only $23 \%$. Moreover, the change in lumen area correlated more strongly with the change in EEM area than with the change in plaque area. Lesions with an increase in EEM area at follow up (47\% of segments studied) showed no change or an actual gain in lumen area and a reduction in angiographic restenosis $(26 \% \mathrm{v}$

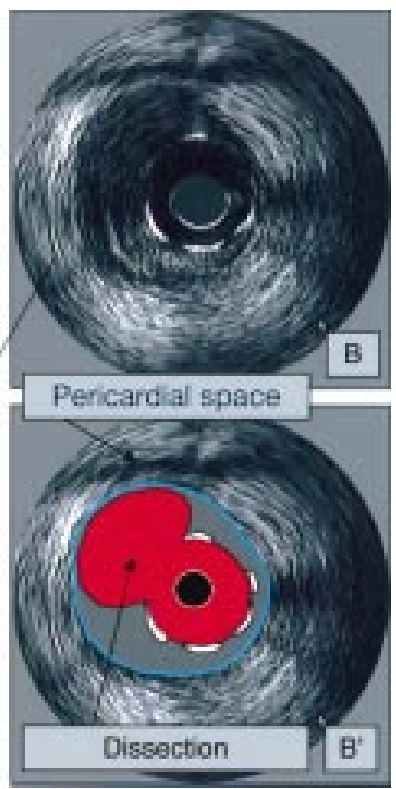

Figure 4 An IVUS interrogation of this segment shows a dissection of the vessel wall extending beyond the stent struts. In the video sequences flow was evident in the dissection cavities. The close proximity to the epicardium is obvious and influenced the decision to intervene.

$62 \%, \mathrm{p}<0.0001)$. These and other studies have established the role of negative remodelling in restenosis after mechanical intervention and explained the success of the concept of additional stenting in an attempt to prevent negative remodelling.

\section{Stenting}

IVUS has provided key insights into the reduction of restenosis rate observed with coronary stenting in comparison to balloon angioplasty. In serial IVUS studies of stented coronary segments, no significant change occurred in the area bound by stent struts, indicating that stents can resist the arterial remodelling process and that stent restenosis is primarily caused by neointimal proliferation. The prevention of negative remodelling, combined with the greater initial lumen expansion with stenting, results in a lower net restenosis rate.

However, during the initial clinical experience with stent implantation, acute stent thrombosis limited application to a relatively narrow subset of patients and required routine use of warfarin. Here, again, IVUS imaging played a pivotal role in optimising clinical results. The pioneering observations of
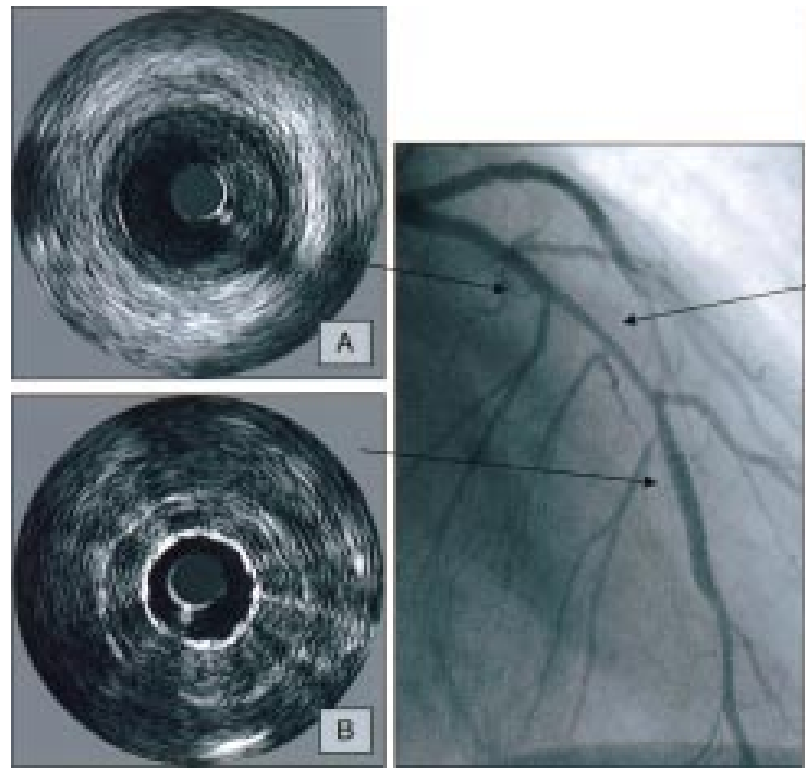

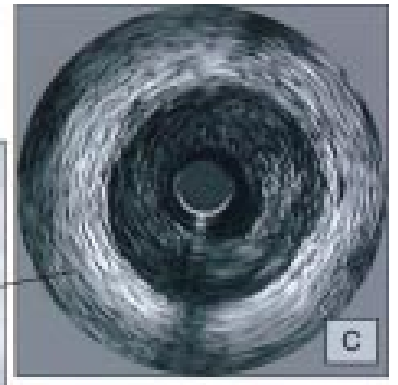

Figure 5 In panel A, the left anterior descending (LAD) artery appears normal on the angiogram, yet on IVUS there is a clear crescent of soft atheromatous plaque. Similarly in panels $C$ and $C^{\prime}$ there is a severe concentric plaque indicating significant luminal narrowing. On angiography, at this point, there is a tubular narrowing as seen by IVUS. In panel $B$, the presence of the covered stent is seen with good and uniform

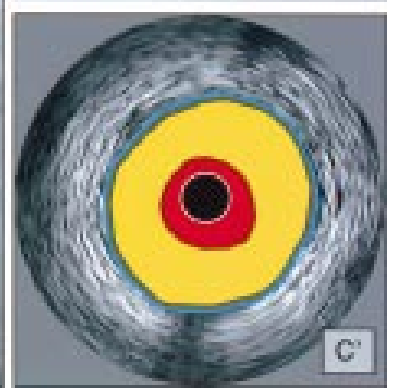

stent expansion and a good angiographic result. 
Colombo and colleagues, based upon IVUS, dramatically changed clinical practice. ${ }^{11}$ These and other investigators showed that stent deployment with conventional balloon pressures resulted in a high incidence of incomplete stent expansion and poor apposition. In a pivotal series, Colombo and colleagues used IVUS imaging to guide high pressure dilatation, achieving full expansion and complete stent apposition in 96\% of 359 consecutive, but non-randomised, patients. Patients with optimal expansion received antiplatelet treatment using aspirin and ticlopidine, but not warfarin. These technical modifications resulted in outstanding clinical outcomes. The incidence of acute and subacute stent thrombosis was less than $1 \%$, and target vessel revascularisation for symptomatic restenosis at six months was 13\%.

Thereafter, the concept of high pressure stent deployment disseminated rapidly, and larger trials demonstrated the safety of stent implantation using high pressures and antiplatelet treatment alone (without IVUS guidance). Consistent with these later trials, IVUS is no longer routinely used for stent optimisation. However, as newer interventional approaches are developed, IVUS imaging is routinely applied early in the life cycle of these innovations, often providing crucial insights into the mechanisms of benefit or complications. Accordingly, IVUS imaging is currently playing an important role in understanding the effects of radiation therapy for in-stent restenosis (brachytherapy), and the effects of drug eluting stents.

\section{ATHEROMA BURDEN AND VULNERABILITY}

It is not uncommon to see a young, previously healthy patient present with a massive myocardial infarction as the first manifestation of CAD. Despite great advances in the acute and chronic management of such patients, these cases demonstrate our failure to make the diagnosis of CAD in time to prevent serious complications. Traditional angiographic methods for assessing atherosclerotic disease rely exclusively upon the identification of significant luminal stenoses as a marker for disease burden. However, IVUS and histological studies show that "significant" stenoses represent only a small fraction of the total atheroma burden in patients with CAD (fig 5). ${ }^{12}$ Presumably, patients with major, unheralded coronary events lack sufficient luminal narrowing to result in exertion ischaemia. In this setting, the acute event originates from rupture of a non-stenotic, clinically "silent" atheroma. Epidemiological data strongly support this model-several angiographic studies have documented that the majority of patients with acute myocardial infarction previously had only low grade stenoses at the culprit lesion site.

Because most acute coronary events are initiated from unheralded rupture of such subclinical "vulnerable" plaques, it is not surprising that angiography provides limited predictive value in identifying the risk of subsequent coronary events. ${ }^{13}$ Currently, the best predictors of future events are established clinical and biochemical risk factors including age, sex, history of diabetes mellitus, hyperlipidemia, family history, and inflammatory markers. However, intracoronary ultrasound offers significant potential as a means to identify and characterise "non-stenotic" coronary atheroma burden, and plaque vulnerability. This approach may allow development of new strategies for interventions (medical or mechanical) by targeting atheromas at risk of rupture. Two strategies are being actively pursued: the assessment of focal plaque vulnerability and quantification of diffuse overall atheroma burden.

\section{Focal plaque vulnerability}

Plaque vulnerability describes the tendency of atherosclerotic lesions to cause atherothrombotic complications. It is well

\section{Key points}

- Tomographic imaging of coronary arteries with intravascular ultrasound (IVUS) demonstrates that simultaneous assessment of lumen and vessel wall can provide important insights into atherosclerotic lesion development and significance

- Important principles described with IVUS-diffuse disease distribution and arterial remodelling-apply to native coronary artery disease (CAD) and lesion response during percutaneous coronary interventions

- The insights previously obtained with IVUS can be applied during interventional treatment of significantly stenotic lesions

- The examination of mildly stenotic lesions may allow an assessment of disease burden and plaque vulnerability and may become important for CAD prevention

established that most acute coronary syndromes are caused by the sudden rupture or superficial erosion of an atherosclerotic plaque. ${ }^{12}$ The histology of these unstable plaques often reveals a lipid laden atheroma with a thin fibrous cap. A cascade of inflammatory processes probably plays a central role in the development and rupture of these lesions. ${ }^{12}{ }^{13}$ IVUS can demonstrate certain morphologic characteristics associated with plaque instability, including the necrotic, lipid rich core, the fibrous cap, and plaque rupture (fig 2). ${ }^{14}{ }^{15}$

More recently, studies have demonstrated a strong association between positive remodelling (enlargement of the EEM) and an unstable clinical presentation (acute myocardial infarction or unstable angina). We examined 85 patients with unstable and 46 patients with stable coronary syndromes using IVUS and found positive remodelling significantly more frequent in unstable than in stable lesions (51.8\% v 19.6\%), while negative remodelling was more frequent in stable lesions $(56.5 \% \quad v 31.8 \%)(\mathrm{p}=0.001) .{ }^{9}$ It is an attractive hypothesis that atheroma inflammation associated with lipid deposition, characteristic of unstable lesions, causes both plaque rupture and vessel expansion. A fibrotic response associated with plaque healing may be associated with negative remodeling. ${ }^{16}$

\section{Diffuse overall plaque burden}

Recent studies demonstrate that plaque vulnerability, plaque rupture, and subsequent plaque stabilisation are highly dynamic and widespread processes. ${ }^{17}$ Subclinical plaque rupture and subsequent healing frequently occur during the development of atherosclerotic lesions and may be a common mode of disease progression. It is therefore an attractive hypothesis that the total plaque burden may be related to the propensity to develop morbid complications of CAD. This is supported by the experience with calcium quantification using computed tomography. Although calcium scoring only quantitates the calcified component of the overall plaque burden, a correlation between the calcium score and clinical events has been consistently demonstrated. In addition, pharmacological studies have suggested a decreased rate of calcium score progression during lipid lowering treatment. ${ }^{18}$

IVUS has great potential as a means to quantify the overall extent of atherosclerotic disease burden. IVUS imaging in patients with limited angiographic CAD typically demonstrates a large disease burden, often showing atherosclerosis in every cross section. Results from a serial IVUS study with measurements of plaque burden as the primary efficacy parameter have recently been reported. ${ }^{19}$ Large serial IVUS 
studies are currently underway examining the effects of different lipid lowering regimens and alternative antihypertensive agents on plaque burden. The results from these studies may further define the role of IVUS in the assessment of $\mathrm{CAD}$ progression or regression and the observation of the transition from stable "silent" disease to acute coronary syndromes.

IVUS represents the first tomographic coronary image technique allowing the assessment of the intramural structures of the vessel wall. However, non-invasive imaging modalities, in particular multi-slice computed tomography and magnetic resonance imaging, are under development for clinical coronary artery imaging. ${ }^{20}$ These non-invasive techniques have particular appeal in a setting of prevention, where the invasive nature of IVUS is a distinct disadvantage. The future cardiologist will use a broadened diagnostic armamentarium to identify a wide range of obstructive and nonobstructive forms of CAD. In addition to the identification and treatment of highly stenotic lesions, which already cause clinical disease, new emphasis will be placed on the identification of "silent" non-obstructive lesions in accordance with the Virschow triad. The systemic and local treatment of these developing lesions has the potential to prevent $\mathrm{CAD}$ progression and avoid morbid complications such as acute coronary syndromes.

\section{CONCLUSION}

The above discussion shows that tomographic imaging of coronary arteries with IVUS has significantly influenced our understanding of the pathophysiology and treatment of CAD. The comparison to angiography explains the complementary role of tomographic imaging of lumen and vessel wall and is exemplified in figs 3, 4, and 5. In this young patient with precocious CAD, IVUS guided the therapeutic, interventional approach of the non-obstructive left anterior descending artery lesion. On the other hand IVUS allowed surprising insights into the disease process by showing extensive, diffuse disease in an angiographically relatively unsuspicious coronary segment.

We believe that tomographic coronary imaging with IVUS and emerging non-invasive modalities can contribute to an early diagnosis of subclinical CAD. It is an attractive hypothesis that this information could lead to improved preventive strategies.

\section{Authors' affiliations}

P Schoenhagen, S E Nissen Department of Cardiology, The Cleveland Clinic Foundation, Cleveland, Ohio, USA

\section{REFERENCES}

1 Mintz GS, Nissen SE, Anderson WD, et al. American College of Cardiology clinical expert consensus document on standards for acquisition, measurement and reporting of intravascular ultrasound studies (IVUS). J Am Coll Cardiol 2001;37:1478-92.

- This ACC/AHA guideline paper gives a complete overview over procedural details of IVUS.
2 Nissen SE, Yock P. Intravascular ultrasound: novel pathophysiological insights and current clinical applications. Circulation 2001;103:604-16.

- This review article summarises clinical applications of IVUS.

3 Glagov S, Weisenberg E, Zarins C, et al. Compensatory enlargement of human atherosclerotic coronary arteries. N Engl J Med 1987;316: $1371-5$

- This seminal article describing arterial remodelling in human coronary arteries has changed our understanding of CAD.

4 Tuzcu EM, Kapadia SR, Tutar E, et al. High prevalence of coronary atherosclerosis in asymptomatic teenagers and young adults: evidence from intravascular ultrasound. Circulation 2001;103:2705-10.

- This article describes the frequent presence of atherosclerotic lesions in young persons who died from non-cardiovascular causes. 5 Mintz GS, Painter JA, Pichard AD, et al. Atherosclerosis in angiographically "normal" coronary artery reference segments: an intravascular ultrasound study with clinical correlations. J Am Coll Cardiol 1995;25: 1479-85.

6 Tuzcu EM, Hobbs RE, Rincon G, et al. Occult and frequent transmission of atherosclerotic coronary disease with cardiac transplantation: insights from intravascular ultrasound. Circulation 1995;91:1706-13.

7 Losordo DW, Rosenfield K, Kaufman J, et al. Focal compensatory enlargement of human arteries in response to progressive atherosclerosis: in vivo documentation using intravascular ultrasound. Circulation 1994;89:2570-7.

8 Pasterkamp G, Wensing PJ, Post M, et al. Paradoxical arterial wall shrinkage may contribute to luminal narrowing of human atherosclerotic femoral arteries. Circulation 1995:91:1444-9.

9 Schoenhagen P, Ziada K, Kapadia SR, et al. Extent and direction of arterial remodeling in stable versus unstable coronary syndromes: an intravascular ultrasound study. Circulation 2000;101:598-603.

- One of the first articles describing the association between direction of remodelling and clinical presentation.

10 Mintz GS, Kent KM, Pichard AD, et al. Contribution of inadequate arterial remodeling to the development of focal coronary artery stenoses: an intravascular ultrasound study. Circulation 1997;95:1791-8.

11 Colombo A, Hall P, Nakamura S, et al. Intracoronary stenting without anticoagulation accomplished with intravascular ultrasound guidance. Circulation 1995;91:1676-88.

- This clinical series influenced the contemporary practice of stenting

12 Ross R. The pathogenesis of atherosclerosis: a perspective for the 1990's. Nature 1993;362:801-9.

- Classical review of CAD which changed the concept of this disease.

13 Libby P. Current concepts of the pathogenesis of the acute coronary syndromes. Circulation 2001;104:365-72.

- A concise review of current concepts about acute coronary syndromes.

14 Yamagishi M, Terashima M, Awano K, et al. Morphology of vulnerable coronary plaque: insights from follow-up of patients examined by intravascular ultrasound before an acute coronary syndrome. J Am Coll Cardiol 2000;35:106-11.

- The first prospective IVUS study defining characteristics of mildly stenotic lesions which subsequently cause acute coronary syndromes.

15 von Birgelen C, Klinkhart W, Mintz GS, et al. Size of emptied plaque cavity following spontaneous rupture is related to coronary dimensions, not to the degree of lumen narrowing. A study with intravascular ultrasound in vivo. Heart 2000;84:483-8.

16 Burke AP, Kolodgie FD, Farb A, et al. Morphological predictors of arterial remodeling in coronary atherosclerosis. Circulation 2002; 105:297-303.

17 Goldstein JA, Demetriou D, Grines CL, et al. Multiple complex coronary plaques in patients with acute myocardial infarction. N Engl J Med 2000;343:915-22.

- This article describes the frequent finding of additional complex angiographic lesions distant from the culprit lesions in patients presenting with acute myocardial infarction.

18 Callister TQ, Raggi P, Cooil B, et al. Effect of HMG-CoA reductase inhibitors on coronary artery disease by electron-beam computed tomography. N Engl J Med 1998;339:1972-8.

19 Schartl M, Bocksch W, Koschyk DH, et al. Use of intravascular ultrasound to compare effects of different strategies of lipid-lowering therapy on plaque volume and composition in patients with coronary artery disease. Circulation 2001;104:387-92.

20 Schroeder S, Kopp AF, Baumbach A, et al. Non-invasive characterization of coronary lesion morphology by multi-slice computed tomography: a promising new technology for risk stratification of patients with coronary artery disease. Heart 2001;85:576-7. 